\title{
Mechanisms of Disease
}

\section{Therapeutic implications of diastolic dysfunction in heart failure}

\author{
John W. Dean and Philip A. Poole-Wilson \\ Department of Cardiac Medicine, National Heart \& Lung Institute, Dovehouse Street, London SW3 6LY, \\ UK
}

'If an old man's heart relaxes slowly, his capacity for physical exertion is thus limited ... even though the systolic contractions were still like those of youth'. ${ }^{1}$

\section{History}

The idea that diastolic dysfunction may give rise to symptoms of cardiac disease independent of any impairment of systolic function is not new, and was appreciated centuries ago. ${ }^{2}$ Traditional definitions of heart failure have focused on abnormalities of systolic or pump function of the heart, emphasizing the failure of cardiac output to meet the metabolic requirements of the body at peak workload. The heart has both systolic and diastolic functions and disturbance of either may produce heart failure. Some of the symptoms and signs of heart failure relate more directly to diastolic function of the ventricles rather than the inotropic state.

\section{Definitions}

The precise timing of the normal phases of the cardiac cycle has changed through the ages, ${ }^{2}$ but the current widely recognized clinical definition is that of Wiggers. ${ }^{3}$ Diastole starts at the moment of closure of the aortic valve and ends when the mitral valve closes. Diastole may be further subdivided into four phases (Figure 1). Ventricular filling, which occurs during diastole, is a complex process and the term 'diastolic dysfunction', much like its systolic counterpart 'contractility', has grown to include abnormalities extending from the fundamental properties of the myocardium to changes in the loading conditions and measured parameters such as peak filling rate.

Correspondence: Professor P.A. Poole-Wilson, M.D., F.R.C.P

Received: 8 June 1990

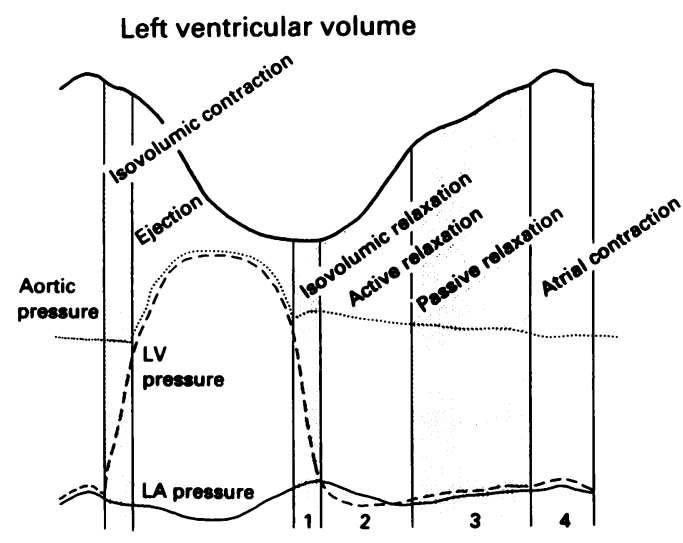

Figure 1 Phases of the cardiac cycle. 1-Isovolumic relaxation time, from aortic valve closure to mitral valve opening. 2-Active relaxation (rapid filling phase), from mitral valve opening to onset of plateau of left ventricular (LV) volume curve. 3-Passive relaxation (slow filling period or diastasis), from onset of plateau to the start of atrial systole. 4-Atrial contraction, from start of left atrial (LA) systole to closure of mitral valve.

Systolic failure may arise because of alterations in the geometry of the heart (shape, size, cell slippage), incoordinate contraction, lack of muscle or a defect of pressure development and fibre shortening. Systolic failure is usually manifest as a reduced ejection fraction or cardiac output.

Diastolic dysfunction may be defined as restriction to filling of one or both ventricles leading to a high atrial pressure, pulmonary or systemic venous hypertension and congestion. In pure systolic left ventricular failure (Figure 2, upper panel) the end systolic pressure-volume curve is shifted down and to the right. In order to maintain a normal stroke volume the ventricle dilates and cardiac output is maintained by the Frank-Starling relationship. The restoration of a normal stroke volume is due to a raised diastolic volume but on the normal diastolic pressure-volume curve. In contrast, pure diastolic left ventricular dysfunction (Figure 2, middle panel) does not affect the end 
systolic pressure-volume curve, but there is an upward shift in the diastolic pressure-volume curve, which is also steeper. Thus stroke volume is maintained but at any given volume the diastolic
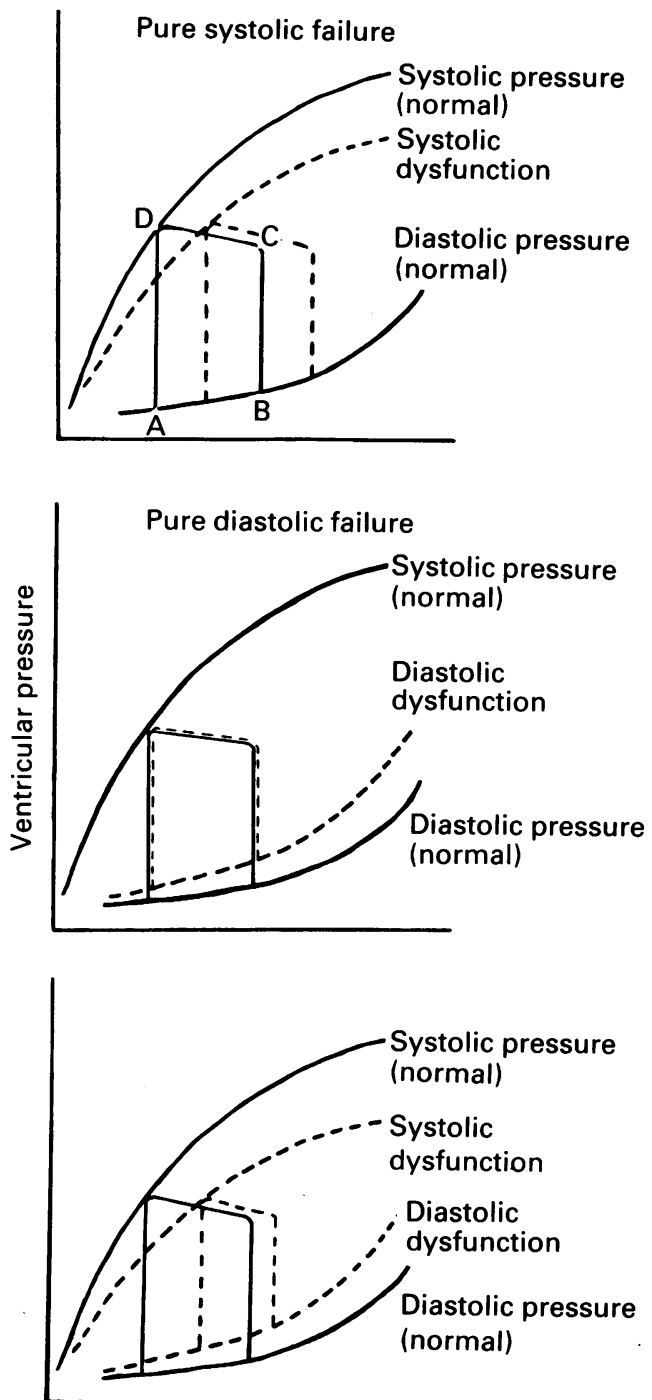

Ventricular volume

Figure 2 Left ventricular pressure-volume loops in normal heart and ventricular failure. The normal pressure-volume loop is represented by the continuous line and the effect of dysfunction (dashed line) during: systole (upper panel), diastole (middle panel) and both systole and diastole (lower panel). Each loop (A-B-C-D) represents one cardiac cycle. In the normal heart the left ventricle fills from the end of isovolumic relaxation (A) until the end of diastole (B). The pressure rise from B to C occurs during isovolumic contraction. Ventricular ejection occurs from $C$ to the end of systole (D), while the fall in pressure from $D$ to $A$ represents isovolumic relaxation. pressure is higher. More commonly systolic and diastolic dysfunction coexist (Figure 2, lower panel) implying both contractile failure and reduced ventricular compliance.

\section{Aetiology}

Diastolic heart disease may be a consequence of structural or functional abnormalities. Structural abnormalities may be due to external compression such as constrictive pericarditis, and tamponade, obstruction to ventricular filling such as mitral or tricuspid stenosis, or atrial myxoma, or may be intramyocardial from amyloidosis or fibrosis giving a stiff or non-compliant ventricle. Functional abnormalities may be myocardial in origin, such as those seen in ischaemia, or due to a haemodynamic abnormality. ${ }^{4}$ Some conditions, such as ventricular hypertrophy, may exhibit both structural and functional abnormalities.

Not all myocardial disease processes are global. Ischaemia due to coronary artery disease, for instance, is almost invariably non-uniform and may produce changes in the time course of contraction and relaxation causing regional myocardial asynchrony. This can have a profound effect on diastolic function of the heart even in the absence of significant changes in inotropic state or loading conditions. ${ }^{5}$ Other less easily defined factors may also play a role in diastolic disease. The pericardium may act as a restraint so that when there is an acute rise in right ventricular pressure and volume the pericardium is stretched, transmitting this increased pressure to the left ventricle. ${ }^{6,7}$ The aortic root may impinge on the left ventricular cavity to restrict filling, ${ }^{8}$ and changes in diastolic arterial pressure may affect ventricular compliance by an 'erectile effect' depending on the degree of coronary turgor.'

The treatment of structural limitation of ventricular filling, such as a constricting pericardium, is to relieve the obstruction wherever possible. It is the therapeutic implications of functional diastolic disease that give rise to controversy and it is on this that this article will concentrate.

\section{Clinical findings}

Several studies have suggested that many patients with the clinical features of chronic heart failure may have normal or near normal systolic function. Using radionuclide angiography, Dougherty et $a l .{ }^{10}$ found a normal ejection fraction $(>45 \%)$ in $36 \%$ of 188 patients with heart failure, Soufer $e t$ al. ${ }^{11}$ found $42 \%$ of 74 patients with similar values, while the VeHeFT study ${ }^{12}$ found that $13 \%$ of 623 patients who were studied had an ejection fraction 
$>45 \%$. Although these studies are widely quoted as evidence that it is possible to have heart failure with normal systolic function the evidence is not convincing, since the ejection fraction is affected by the loading conditions and thus may not necessarily indicate normal inotropic state. Furthermore, the patients were studied at rest and the measured indices may change on exercise. Many patients were taking drugs (such as digoxin and diuretics) which may affect the results. More importantly, the objective finding of a normal ejection fraction in a patient with a clinical diagnosis of heart failure should raise doubt about the accuracy of the diagnosis, the symptoms and signs may have been misinterpreted..$^{13} \mathrm{~A}$ patient with dyspnoea and ankle oedema may have chronic heart failure, but may also have asthma and varicose veins!

Despite these criticisms, many patients with heart failure are disabled to a greater extent than their pump function alone would seem to suggest they should be, and even those patients with severely impaired systolic function usually have coexistent diastolic dysfunction. But whether the diastolic dysfunction plays an important role in producing symptoms in the majority of patients remains open to question. There appears to be little relationship between diastolic function either at rest or on exercise to symptoms limiting exercise. ${ }^{14}$

\section{Cellular mechanisms}

The cellular mechanisms responsible for diastolic dysfunction appear to relate to impaired myocyte calcium handling. ${ }^{15}$ Release of calcium from the sarcoplasmic reticulum is responsible for the initiation of contraction, and this appears unaffected in heart failure whereas reuptake (an active process necessary for relaxation to occur) is delayed. There is an associated reduction in sarcoplasmic reticulum calcium ATPase activity. Cyclic AMP deficiency due either to beta-receptor down regulation or altered regulation of adenylate cyclase would lead to impaired diastolic relaxation due to inactivity of the calcium regulatory protein phospholamban, or reduced slow channel conductance of calcium. In this regard agents that increase cyclic AMP such as milrinone (which would inhibit breakdown by phosphodiesterase inhibition) or forskolin (an adenylate cyclase activator) can improve diastolic function in laboratory and clinical studies. ${ }^{16-18}$ However, it is uncertain whether the effects in man are due to a secondary vasodilatation rather than a direct effect on the myocardium.

Since more energy is required for calcium uptake rather than for the initiation of contraction, the reduction in cyclic AMP needed to impair relaxation is less than that required to impair contractility
- thus diastolic abnormalities occur earlier and are a more sensitive marker of a deficient energy state in heart failure. In acute ischaemia due to coronary occlusion the profoundly depressed systolic function overshadows any diastolic dysfunction, but in demand ischaemia (e.g., pacing tachycardia or exercise in the presence of restricted coronary blood flow) the diastolic abnormalities may predominate. $^{19}$

\section{Clinical recognition}

How is it possible to identify those patients with symptoms and signs of heart failure who have predominantly diastolic dysfunction? The aetiology is most likely to be ischaemia due to coronary artery disease or left ventricular hypertrophy most commonly due to chronic hypertension. ${ }^{11}$ Clinically and radiographically the heart size is often normal or only slightly enlarged, echocardiography may show thickening of the left ventricular wall but with relatively well preserved systolic wall motion. Reduced diastolic posterior aortic wall motion, as well as a variety of other diastolic abnormalities, have been described. ${ }^{20}$ Doppler studies of ventricular filling patterns during early diastole ( $E$ velocity) and late diastole (A velocity) can be used to calculate the $\mathrm{E} / \mathrm{A}$ ratio as a single index of diastolic performance. Much emphasis has been placed on depression of the $E / A$ ratio or of peak filling rates as a manifestation of impaired ventricular compliance, but a variety of patterns (including normality) are possible in the presence of diastolic dysfunction. ${ }^{21}$ Likewise, radionuclide techniques may show a reduced peak filling rate and in some instances an increased contribution of atrial contraction to left ventricular filling. ${ }^{22}$ Much confusion has arisen because of the expression of peak filling rates in terms of the end diastolic volume of the left ventricle. Figure 3 shows quite clearly that patients with dilated ventricles will have an apparently low peak filling rate compared to controls when this is expressed as end diastolic volumes per second, although the peak filling rate in $\mathrm{ml} / \mathrm{s}$ is virtually identical in all 3 groups.

\section{Management}

\section{General}

The management of patients with heart failure and predominantly diastolic dysfunction should include both symptomatic as well as preventative treatment. Traditional therapy for heart failure may not always be appropriate, indeed, inotropes and arterial vasodilators may prove harmful to such patients. General measures are important, 


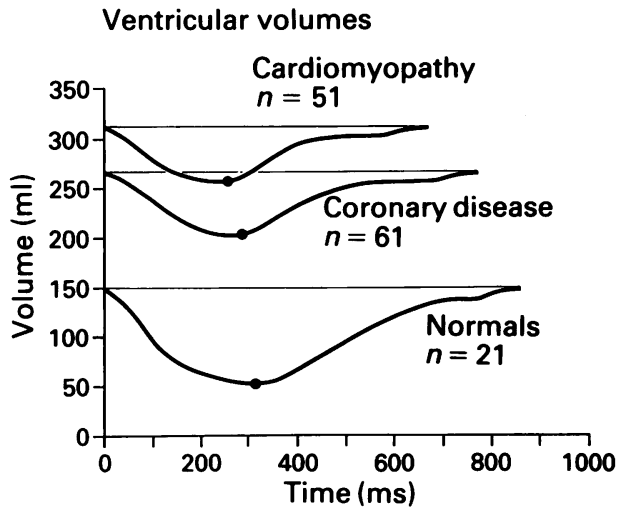

Figure 3 Left ventricular volume-time curves measured by radionuclide ventriculography for patients with dilated cardiomyopathy, coronary artery disease and controls (from Ref. 22). Based on Lavine et al. Int J Cardiol 1985, 8: 423.

hypertension should be treated early to prevent the development of left ventricular hypertrophy. If this already exists, progression should be prevented and regression encouraged by appropriate therapy. The acute increase in left ventricular diastolic pressure that occurs during ischaemia ${ }^{23}$ can be treated with nitrates. A more rational goal, however, would be prevention of ischaemia and its consequences both acute (impaired left ventricular relaxation and compliance) and chronic (fibrosis and scar). Thus beta-blockers, calcium antagonists and nitrates all play their role in the treatment and prevention of diastolic dysfunction.

Slowing of relaxation usually blunts the rapid early diastolic left ventricular filling, and patients become more dependant on atrial systole for adequate filling. Such patients will tolerate loss of a correctly timed atrial contraction poorly and maintenance of normal atrio-ventricular synchrony would be a therapeutic goal. Cardioversion of atrial fibrillation should be performed wherever possible and if heart block develops dual chamber pacing should be considered.

\section{Diuretics}

A major goal of treatment should be to reduce pulmonary and systemic venous pressures and thereby relieve congestion. Diuretics are effective but the dose required may be small, because the stiff ventricle has a steep diastolic pressure volume curve and large changes in pressure can be achieved with only modest changes in volume.

\section{Vasodilators}

Venodilators such as nitrates improve exercise capacity in heart failure and reduce left ventricular end diastolic pressure, perhaps by reducing right ventricular end diastolic pressure and consequently the pericardial restraint on the left ventricle, although most venodilators also reduce arteriolar resistance and thus afterload to a lesser extent. Nitrates have the added advantage of providing anti-ischaemic therapy.

Angiotensin converting enzyme (ACE) inhibitors such as captopril reduce left ventricular end diastolic pressure to a greater degree than may be explained by their effect on venous capacitance or ventricular volume, once again this may be due to a reduction in afterload, but it is known that angiotension can impair ventricular relaxation suggesting that ACE inhibition may have an additional direct myocardial effect.

\section{Beta-adrenoreceptor agonists}

Beta agonists probably improve diastolic function by enhancing calcium reuptake by the sarcoplasmic reticulum facilitating relaxation. However, tolerance does develop so these drugs have little if any role in the long term management of chronic heart failure. The beta ${ }_{1}$ partial agonist xamoterol, which has been shown to improve effort tolerance in heart failure, ${ }^{24}$ may be an exception, tolerance does not appear to develop and long term beneficial effects on left ventricular end diastolic pressure with no change in left ventricular volume have been demonstrated..$^{25}$ This may be because the drug acts as a beta blocker rather than an agonist in failing human heart muscle. ${ }^{26}$

\section{Betablockers}

Beta blockers have been shown to improve symptoms in patients with heart failure although the mechanism is unclear. ${ }^{27}$ Long term beta blocker therapy appears to restore beta adrenergic sensitivity which is usually lost in chronic heart failure, possibly due to an increase in beta receptor density. ${ }^{28}$ Perhaps also the reduction in heart rate induced by these drugs prolongs the filling time and this may be of particular benefit to patients with a resting tachycardia especially when filling is prevented by 'diastolic' mitral or tricuspid regurgitation. ${ }^{4}$ In patients with ischaemia beta blockers are energy sparing, reducing myocardial oxygen requirements. Beta blockers may also prevent damage which may occur in the heart due to increased plasma catecholamines.

\section{Calcium antagonists}

Although calcium channel blockers such as verapamil and nifedipine are of value in the treatment of hypertrophic cardiomyopathy ${ }^{29,30}$ there is little evidence to support their widespread use in chronic 
heart failure. Any improvement in diastolic function after calcium channel blockade appears to be related to the vasodilator effects of these drugs, indeed, the decrease in cytosolic calcium produced by these drugs impedes myocardial relaxation unless sympathetic reflexes are activated..$^{31,32}$

\section{Conclusion}

When the patient with heart failure receives effective treatment both the systolic and diastolic state of the ventricles may be improved and the patient becomes less symptomatic. Whether short-

\section{References}

1. Henderson, Y. Volume changes of the heart. Physiol Rev 1923, 3: 165-208.

2. Brutsaert, D.L. \& Sys, S.U. Relaxation and diastole of the heart. Physiol Rev 1989, 69: 1228-1315.

3. Wiggers, C.J. Studies on the consecutive phases of the cardiac cycle. Am J Physiol 1921, 56: 415-459.

4. Ng, K.S.K. \& Gibson, D.G. Impairment of diastolic function by shortened filling period in severe left ventricular disease. Br Heart J 1989, 62: 246-252.

5. Brutsaert, D.L. Non-uniformity: a physiologic modulator of contraction and relaxation of the normal heart. $J \mathrm{Am}$ Coll Cardiol 1987, 9: 341-348.

6. Bemis, C.E., Serur, J.R., Borkenhagen, D., Sonnenblick, E.H. \& Urschel, C.W. Influence of right ventricular filling pressure on left ventricular pressure and dimension. Circ Res 1974, 34: 498-507.

7. Junemann, M., Smiseth, D.A., Refsum, H. et al. Quantification of effect of pericardium on left ventricular pressure-volume relation in dogs. Am J Physiol 1987, 252: H963-H968.

8. Brodie, B.R., Grossman, W., Mann, T. \& McLaurin, L. Effects of sodium nitroprusside on left ventricular pressurevolume relations. J Clin Invest 1977, 59: 59-68.

9. Salisbury, P.F., Cross, C.E. \& Rieben, T.A. Influence of coronary artery pressure upon myocardial elasticity. Circ Res 1960, 8: 794-802.

10. Dougherty, A.H., Naccarelli, G.V., Gray, E.L., Hicks, C.H. \& Goldstein, R.A. Congestive heart failure with normal systolic function. Am J Cardiol 1984, 54: 778-782.

11. Soufer, R., Wohlgelernter, D., Vita, N.A. et al. Intact systolic left ventricular function in clinical congestive heart failure. Am J Cardiol 1985, 55: 1032-1036.

12. Cohn, J.N. \& Johnson, G. Heart failure with normal ejection fraction: The V-HeFT study. Circulation 1990, 81 (Suppl III): 48-53.

13. Marantz, P.R., Robin, J.N., Wassertheil-Smoller, S. et al. The relationship between left ventricular systolic function and congestive heart failure diagnosed by clinical criteria. Circulation 1988, 77: 607-612.

14. Lipkin, D.P. \& Poole-Wilson, P.A. Symptoms limiting exercise in chronic heart failure. $\mathrm{Br}$ Med J 1986, 292: 1030-1031.

15. Gwathmey, J.K., Copelas, L., MacKinnon, R., Schoen, F.J., Grossman, W. \& Morgan, J.P. Abnormal intracellular calcium handling in myocardium from patients with endstage heart failure. Circ Res 1987, 61: 70-76.

16. Feldman, M.D., Copelas, L., Gwathmey, J.K. et al. Deficient production of cyclic AMP: pharmacologic evidence of an important cause of contractile dysfunction in patients with end-stage heart failure. Circulation 1987, 75: 331-339. ness of breath is relieved by a direct effect of the therapeutic intervention on intrinsic myocardial function or by an indirect effect due to modification of loading conditions is not as important to the patient as the fact that these distressing symptoms are minimized. From a scientific perspective and perhaps from a prognostic standpoint, the precise mechanism underlying these changes is of interest and may be important. Patients with heart failure need to be fully investigated with echocardiography. The presence of systolic or diastolic dysfunction does materially affect the approach to treatment.

17. Bristow, M.R., Ginsburg, R., Umans, V. et al. Beta ${ }_{1-}$ and beta $_{2}$-adrenergic receptor subpopulations in nonfailing and failing ventricular myocardium: coupling effects of both receptor subtypes to muscle contraction and selective beta $a_{1}-$ receptor downregulation in heart failure. Circ Res 1986, 59: 297-309.

18. Monrad, E.S., McKay, R.G., Baim, D.S., Heller, G.V., Royal, H.D. \& Grossman, W. Improvement in indices of diastolic performance in patients with congestive heart failure treated with milrinone. Circulation 1984, 70: 1030- 1037.

19. Apstein, C.S. \& Grossman, W. Opposite initial effects of supply and demand ischemia on left ventricular diastolic compliance: The ischemia-diastolic paradox. $J$ Mol Cell Cardiol 1987, 19: 119-128.

20. Caidahl, K., Eriksson, H., Hartford, M. et al. Dyspnoea of cardiac origin in 67 year old men: (2) relation to diastolic left ventricular function and mass. Br Heart J 1988, 59: 329-338.

21. Stoddard, M.F., Pearson, A.C., Kern, M.J., Ratcliff, J., Mrosek, D.G. \& Labovitz, A.J. Left ventricular diastolic function: comparison of pulsed Doppler echocardiographic and hemodynamic indexes in subjects with and without coronary artery disease. J Am Coll Cardiol 1989, 13: 327-336.

22. Lavine, S.J., Krishnaswami, V., Shreiner, D.P. \& Amidi, M Left ventricular diastolic filling in patients with left ventricular dysfunction. Int $J$ Cardiol 1985, 8: 423-436.

23. Levy, R.D., Shapiro, L.M., Wright, C., Mockus, L. \& Fox K.M. Haemodynamic response to myocardial ischaemia during unrestricted activity, exercise testing, and atrial pacing assessed by ambulatory pulmonary artery pressure monitoring. Br Heart J 1986, 56: $12-18$.

24. The German and Austrian xamoterol study group: Doubleblind placebo-controlled comparison of digoxin and xamoterol in chronic heart failure. Lancet 1988, i: 489-493.

25. Pouleur, H., Van Eyll, C., Hanet, C., Cheron, P., Charlier, A.A. \& Rousseau, M.F. Long term effects of xamoterol on left ventricular diastolic function and late remodelling: a study of patients with anterior myocardial infarction and single vessel disease. Circulation 1988, 77: 1081-1089.

26. Bohm, M., Mittman, C., Schwinger, R. \& Erdmann, E. Xamoterol and the failing heart. Lancet 1989, ii: 1213-1214.

27. Swedberg, K., Waagstein, F., Hjalmarson, A. \& Wallentin, I. Beneficial effects of long term beta-blockade in congestive cardiomyopathy. Br Heart J 1980, 44: 117-123. 
28. Heilbrunn, S.M., Shah, P., Bristow, M.R., Valantine, H.A., Ginsburg, R.\& Fowler, M.B. Increased beta-receptor density and improved hemodynamic response to catecholamine stimulation during long-term metoprolol therapy in heart failure from dilated cardiomyopathy. Circulation 1989, 79: $483-490$

29. Lorell, B.H., Paulus, W.J., Grossman, W., Wynne, J. \& Cohn, P.F. Modification of abnormal left ventricular diastolic properties in patients with hypertrophic cardiomyopathy. Circulation 1982, 65: 499-507.

30. Bonow, R.O., Rosing, D.R., Bacharach, S.L. et al. Effects of verapamil on left ventricular systolic function and diastolic filling in patients with hypertrophic cardiomyopathy. Circulation 1981, 64: 787-796.
31. Kurnick, P.B., Courtois, M.R. \& Ludbrook, P.A. Effects of nifedipine on intrinsic myocardial stiffness in man. Circulation 1986, 74: 126-134.

32. Walsh, R.A. \& O'Rourke, R.A. Direct and indirect effects of calcium entry blocking agents on isovolumic left ventricular relaxation in conscious dogs. J Clin Invest 1985, 75: 1426-1434. 